\title{
An efficient Multiobjective Dynamic Task Scheduling to Minimize Task Execution Time for Computational Grid
}

\author{
Sophiya Sheikh $\mathbb{1}^{\# 1}$ \\ \#1 Central University of Rajasthan, Bandarsindri, Kishangarh, Ajmer 305817, \\ 9413646471, sophiya.sheikh@gmail.com
}

\section{ABSTRACT}

Scheduling in grid is proven to be NP-complete problem. Grid computing is the name of effective utilization of geographically dispersed resource in order to fulfill the resource requirement of users. Computational grid is a part of grid computing performs compute intensive tasks. Grid is empowered to utilize idle resource power and ensure load balancing among processors. However, Grid performance can still be improved by increased parallelism among task to execute and scheduled task dynamically. In this paper we propose MDTS Multiobjective Dynamic Task Scheduling (MDTS) approach to minimize job execution time and enhance TLP (task level parallelism). The acquired empirical results show that MDTS exponentially increase the idle resource utilization however slightly reduces task execution cost and yield better value of major scheduling parameters (Makespan, Flowtime and Average Utilization). Simulation of proposed approach MDTS is specific to a grid simulation toolkit (Gridsim) to design and evaluate scheduling algorithm.

Corresponding Author: Sophiya Sheikh

\section{INTRODUCTION}

Grid computing refers as distributed computing that works upon the integration and collaboration of geographically-dispersed resources. To manage such a high intensive heterogeneous environment resource allocation should be not only scalable but also highly reliable. The usage of grid computing has been exponentially increasing in science, industries and in various others applications related to working in distributed environment. Computational grid plays a vital role in resource sharing and load balancing among resources. It has become most common and widely adapted technique to solve dynamic and computational problem occurred in scientific and technical areas [1]. Scientific and Engineering applications are now extensively using grid resources to solve their computational intensive problems. A computational grid provides user a platform to execute the compute intensive jobs which cannot be executed at the user's end [2]. In a view of the proper usages of underutilized resources, allocation technique of resources must be efficient as we need to process large no of computational jobs. To enhance performance of resources in decentralized architecture task scheduling needs to be done efficiently. In computational grid task could be either dependent tasks or independent tasks [3]. The independent tasks are called metatask that can be executed in parallel in distributed environment. Expected Time to Compute ETC $[\mathrm{t}][\mathrm{m}]$ matrix is extensively used in metatask scheduling. ETC matrix provides the expected execution time of a task executed on a particular resource. It contains $t$ rows and $\mathrm{m}$ columns which represent tasks and machines respectively [4]. Grid scheduler makes use of this matrix to identify the best resource for scheduling a task. Many tools are available in literature for generating DAG for levalized allocation of task [5]. In this paper we use gridsim simulator to generate ETC matrix. For independent 
scheduling ETC matrix is best suited for calculating and minimizing job execution time. The GridSim toolkit allows modelling and simulation of entities in parallel and distributed computing (PDC), peer to peer computing (P2P) and multi cluster computing for design and implementation of task scheduling algorithms. In grid system resources are heterogeneous in nature that can be aggregated using resource brokers. A resource consist one or more processing elements with shared or distributed memory [6]. Resources are managed by scheduler that could be either time shared or space shared. The processing nodes within a resource can be heterogeneous in terms of processing capability, configuration, and availability. The resource brokers uses different task scheduling algorithms or models for mapping task to resources to achieve both system and user objective.

\section{RELATED WORK}

A novel model for secure grid architecture adapted Artificial Neural Network introduced [7]. Artificial Neural Network module provides a secure task machine mapping to overcome the problems related to machine failure. Estimated time to compute matrix has been used to achieve robustness in computational task[6]. Robustness is important feature in heterogeneous computational grid. It can be achieved by work completion latency. In immediate mode scheduling [9] when job arrives in the system it has to be allocated to the resources. For efficient memory allocation author measured some system parameters like makespan time, flowtime,matching proximity and resource utilization. Braun et al [4] has defined a simulation model to generate ETC matrix. They have generated ETC matrix for all types like inconsistent, semi consistent and consistent. In [10] has designed an algorithm for creating ETC matrices. But problems with this method it does not produce low-task high-machine heterogeneity matrices. They could not generate consistent ETC matrices also. In [11] use a simulation model based on ETC matrix which has been generated for 512 tasks and 16 machines. In [12] has used a scheduling model for QoS parameters based on expected time of tasks on multiple machines. In [13] have proposed a task scheduling policy based on memory availability and introduced a memory based task partition and scheduling.

\section{PROBLEM FORMULATION}

Proposed scheduling strategy Multiobjective Dynamic Task Scheduling works for multiple tasks divided into subtask and allocated dynamically to resources to increase processors capability in MIPS and minimizing task execution time. This section describes various component related to grid optimization parameters, ETC models and speed up resources.

\section{Expected time to compute model}

Capability of task can be calculated in millions instruction and processors capability in millions instruction per second. Expected time to compute matrix compute processing time of resources for each task. ETC $[\mathrm{t}][\mathrm{m}]$ refers as expected time to compute for $\mathrm{t}$ task executed in $\mathrm{m}$ machine. In simulation we are dividing single task into multiple subtask then allocated dynamically to resources. ETC $[\mathrm{t}][\mathrm{m}]$ model is designed to calculate the computing capacity of resources [14].

Why we use it: - ETC model is developed to achieve consistency among resources. Consistency refers to the coherence among execution time of a machine with the rest of the machines that executes a set of task. In order to systematic working in distributive environment in which we need to join multiple machines we need to achieve task level parallelism (TLP).

ETC matrix can be classified in terms of task heterogeneity, machine heterogeneity and computational consistency. Job heterogeneity refers to the variation in execution times for all jobs executed on a given machine. Machine heterogeneity refers to the variation in terms of execution times of all machines for a given job. Based on consistency level we can design ETC matrix as consistent, inconsistent and semi consistent [4]. An ETC matrix is called consistent if, for two machines $\mathrm{m} 1$ and $\mathrm{m} 2$, if $\mathrm{m} 1$ executes a task faster than $\mathrm{m} 2$ then $\mathrm{m} 1$ executes all the jobs faster than $\mathrm{m} 2$. While, in an inconsistent ETC matrix, $\mathrm{m} 1$ machine exeute some job faster than $\mathrm{m} 2$ and some 
job slower than m2. Semi consistent ETC matrices are combination of consistence and inconsistent matrices.

\section{Speed-up for resources}

In this paper our main objective is to utilize resources efficiently. We also need to get speed up in job completion for fixed workload of resources. We also need to set the upper bound of resource capability.

$S_{\text {latency }}(s) \leq \frac{1}{1-p}$

$\lim _{n \rightarrow \infty} \operatorname{Slatency}(s) \frac{1}{1-p}$

Shows that theoretical speedup of the execution of whole task increases with the improvement of utilization of resources in grid system. In proposed approach task $t$ is divide into multiple sub tasks like $\{\mathrm{t} 1, \mathrm{t} 2, \mathrm{t} 3, \ldots ., \mathrm{tn}\}$. Before speed up execution time is denoted by $\mathrm{T}$ and percentage of the execution time of the task with improvement of the resources is denoted by $\mathrm{p}$. And the part of task which is not benefited by the improvement is denoted by (1-p) so we can say that:-

$\mathrm{T}=(1-\mathrm{p}) \mathrm{T}+\mathrm{pT}$

The benefited part of task is $: \frac{p}{s} \mathrm{~T}$

So the theoretical execution time of complete task after improvement of resources is :-

$\mathrm{T}(\mathrm{s})=(1-\mathrm{p}) \mathrm{T}+\frac{p}{\mathrm{~s}} \mathrm{~T}$

So finally the theoretical speedup in the latency of the execution of the whole task at fixed workload, defines as

$\mathrm{S}_{\text {latency }}(\mathrm{s})=\frac{T W}{T(s) W}=\frac{T}{T(s)}=\frac{1}{1-p+\frac{p}{S}}$

Theoretical speedup in latency for task execution is evaluated for different value of $\mathrm{P}$. The speedup is limited by the serial execution of task. Proposed dynamic approach MDTS can enhance speed up of a task by increased parallelism.

\section{Grid Optimization Parameters}

Computational grid has various benefits like Easy demand-driven access to computational resources will improve. Better utilization of idle cycles of processors is necessary when we have a large amount of computational tasks. On basis of either performance or optimization we can enhance the capability of grid. MDTS is the Meta heuristic approach and multi objective algorithm. Task Scheduling is performed dynamically by using $\mathrm{Sj}$ scheduler for each machine $\mathrm{j}$. Several parameters can be considered for performance optimization. Some parameters like minimum completion time, makespan time, flowtime, average utilization and provide optimum scheduling. We can also optimized grid by optimized CPU utilization, throughput, turnaround time, waiting time and response time [14].

In proposed approach we consider makespan time, flowtime and minimum completion time as optimization criteria. If we are able to minimize makespan time, flowtime and job completion time then we can achieve efficient task to resource mapping. Minimization of makespan means scheduler providing efficient task to resource mapping. But makespan time alone is not sufficient for performance optimization. Another considered parameter is flowtime, with minimum value of flowtime we can reduce response time of resources. Flowtime implies time duration between job submissions to job completion. With minimum flowtime we can minimize average response time of resources. 
Makespan time is finishing time of last executed task while Flowtime is total execution time of all tasks. Makespan can be expressed in terms of the completion time of resources.

Makespan $=\max \{$ CompTime $[\mathrm{j}] \mid \mathrm{j} \in$ Machines $\}$

In dynamic and distributed environment batch of tasks allotted to resources, largest machine execution time of task called makespan time. Makespan indicates that maximum time taken by machine $\mathrm{j}$ for task completion.

Minimization of makespan defines as:

MinMakespanTime $=\min \operatorname{sj} \epsilon s c h e d($ Max iєtask Ti) $(2)$

Where Ti denotes completion time of task $\mathrm{j}$. Sched sj is scheduler of all tasks $\mathrm{j}$ further submitted for execution.

Completion time of machine $m$ denotes that execution time of previously allotted task and currently executed task. Ready time is the time taken by previously assigned task to execute. Ready time will add to current task execution time to find completion time. Task completion time should be minimum to enhance CPU throughput and productivity.

Minimum completion time: - A task is divided into multiple jobs and each job is mapped to associate resources based on scheduling algorithm. The MCT is minimum completion time taken by a task to execute.

$\mathrm{MCT}=\min \{$ CompTime$[\mathrm{j}] \mid \mathrm{j} \in$ Machines $\}$

Where task j scheduled by $\mathrm{Sj}$ scheduler.

Flowtime is sum of completion times of all the tasks. It is denoted as finalizes time of all tasks. We are taking multiple tasks in our approach. At the end of execution we combine all these tasks. In proposed approach our task is divided into number of subtask and total execution time of all the sub task refers as flowtime.

Flowtime $=\Sigma\{$ CompTime $[\mathrm{j}] \mid \mathrm{j} \in$ Machines $\}$

Minimization of flowtime defines as:

Min Flowtime $=\min \operatorname{sj} \epsilon s c h e d(\min i \in t a s k ~ T i)$

Where Ti denotes completion time of task $\mathrm{j}$. Sched $\mathrm{sj}$ is scheduler of all tasks $\mathrm{j}$ further submitted for execution.

In grid system machines further divided into resources and resources further divided into processing elements. Sub task can be allotted to the processing elements. To achieve such a high level of optimization, Matching proximity and average utilization of resources should be high. To maximize average utilization we need to increase throughput of system. Average utilization can be referred as:

AverageUtilization $=$ Flowtime/MakespanTime

Value of flowtime and makespan time can be calculated by Eq 2 and Eq 3. 


\section{PROPOSED ALGORITHM}

In grid environment for optimum utilization of resources make span time should be minimized which leads to scheduler assigning the task an efficient way to the resources. Make span time is given for each task and this refers to the task allocation to resources in better way. It is necessary to achieve systems related and user related objectives that we should divide the task and each task have minimum make span time [9]. Apart from make span time we will also considered flow time which is response time from task submission to task completion. By minimization of flow time average response time of grid system can be reduced while by minimizing Makespan jobs will be execute in faster way. In proposed work we are scheduling $\mathrm{n}$ jobs to $\mathrm{m}$ resources.

\section{Parallelised Dynamic Task Scheduling}

Start Execution

Initialize Entities ()

1. Generate grid user //Get list of users who have gridlet to execute

2. Generate Gridlet // Create list of gridlet or task for the grid user

I

Create Gridlet //Create a container to store gridlet

Set PE MIPS rating

Assign parameters id, length, fileSize and outputSize to Gridlet

Set users of this gridlet

Store gridlet into llist

\}

3. Generate Grid Resources

I

Get Machine List // Get list of available machine with id, no of PE and MIPS rating of each PE

Set MIPS ratings for each machine

4. Create Resources // manage to register themselves to GIS entity.

\{

5. while(AvailableResources)

\{

if (Resources Available)

else

Break

Wait for Resources

\}

6. for all available resources do //To get all available resources

Allocate resources to gridlet

end for

7. for all Gridlet do

I

Assign each gridlet to random grid resource entity

Allocate each gridlet to resource

Waiting to get gridlet back from resources

Store the receive gridlet into gridlet list

\}

end for

8. Get processing cost for each gridlet

\}

9. Start Simulation

10. Get GridletList for Each users

I

Get resource for each gridlet

Create list of gridlet for each user

\}

11. for all gridlet do

\{

12. Print gridletId, ResourceId, ResourceallocationCost

13. Compute Makespan as per eq 1

14. Compute Flowtime as per eq 4 
15. Compute Average Utization as per eq 6

\}

end for

Task $t$ executes on $m$ machine so we need to compute expected time of all task to execute. Expected time to compute (ETC) model simply defines as ETC $[\mathrm{t}][\mathrm{m}]$ that means task $\mathrm{t}$ will execute on $\mathrm{m}$ machine by certain amount of time[2]. Each user has some job or gridlet will take some time to execute. In proposed algorithm we simulate machines that execute gridlets of users. We need to see first that whether the requested resource is available or not. Resources need to register themselves for GIS entity.

\section{SIMULATION STUDY}

Simulation has been performed on Gridsim toolkit. The Gridsim toolkit based on java based discrete events which can simulate millions of resources and thousands of users. The toolkit is designed to simulate the heterogeneous grid environment. Resources are managed by scheduler that could be either time shared or space shared. The simulation can be performed at user level or processors level. It also simulates multiple resources and task which is corresponding to multiple users. Apart from this load balancing among resources, mapping multiple task to resources, executing task from multiple resources are its key benefits [3].

\section{Resource Model}

In simulation using gridsim we assume that Grid is a collection of various resources. Each resource communicates via communication links and each resource has its own processing speed and computational capability. Normally each resource consists of multiple machines and each machine consists of various processors. Processors have different computing powers and processing capabilities. If task movement is in between two processors than we need to calculate communication cost among processors. While if task movement is in between nodes than we need to calculate inter node communication cost. However in our simulation environment gridsim assume processor communication cost and inter node communication cost as zero. Emphasis is on increase parallelism rather than communication cost.

\section{Task Model}

If we see the simulation from user's perspective we assume multiple users have multiple tasks to execute. Each task is divided into multiple parts and allocated to resources. Task to resource mapping performs by Gridsim simulation toolkit. Each task is independent in nature that can be executed dynamically. Each task needs different execution time as size of task is also different. They may have different input and output size requirements. The processors capability can be calculated in MIPS (millions instructions per second) while task can be presented in millions instructions.

Table 1.

\begin{tabular}{|c|c|c|}
\hline \multicolumn{3}{|c|}{ Resource Specification } \\
\hline Machine & PE & MIPS rating \\
\hline 0 & 4 & 377 \\
\hline 1 & 4 & 377 \\
\hline 2 & 2 & 377 \\
\hline
\end{tabular}

Table 2.

\begin{tabular}{|l|l|}
\hline \multicolumn{2}{|c|}{ Gridlet Parameters } \\
\hline Length & 0 to $50,000 \mathrm{MI}$ \\
\hline File Size & $\begin{array}{l}\text { Varies within this range } \\
100+(10 \% \text { to } 40 \%)\end{array}$ \\
\hline Output Size & $\begin{array}{l}\text { Varies within this range } \\
100+(10 \% \text { to } 50 \%)\end{array}$ \\
\hline Gridlet & 8 to n \\
\hline
\end{tabular}




\section{RESULTS}

Here we are comparing static task scheduling (STS), dynamic task scheduling (DTS), multiobjective dynamic task scheduling (MDTS). In proposed strategy we simulate three resources with id 5, 9 and 13. Number of gridlets can be varying from 8 to $\mathrm{n}$. Each resource have some allocation cost given in MIPS (Millions instruction per second). Results show that if resource allocation is static or resources are allocated in continuous manner then processors capability is low in terms of MIPS. We observed that proposed MDTS provides us high processing capability and task execution rate (MIPS). MDTS enhances resource utilization with minimizing job execution time.

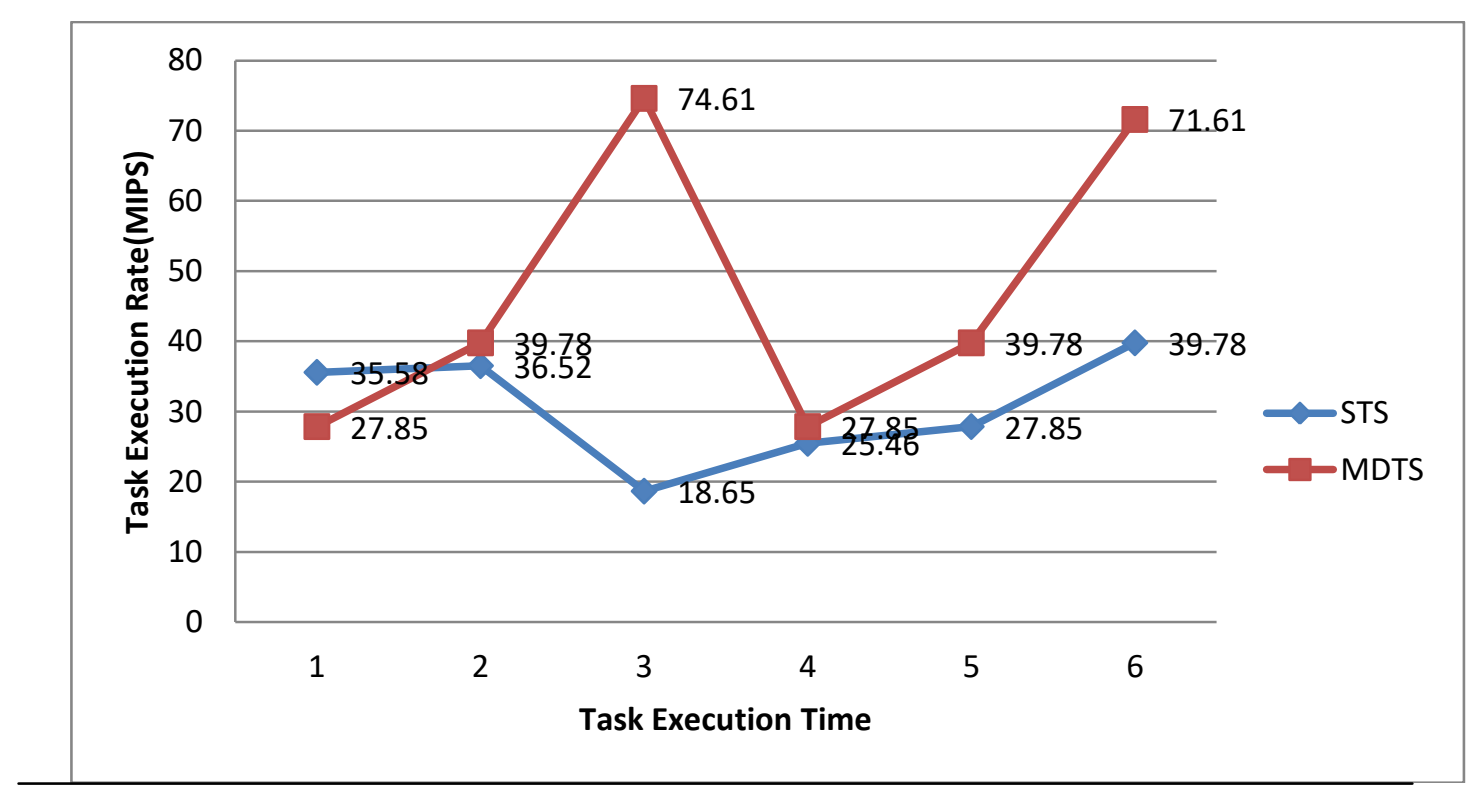

Fig 1.Comparing different approaches in terms of Processing Capability (MIPS)



Fig 2. Makespan for Multiple Heuristics

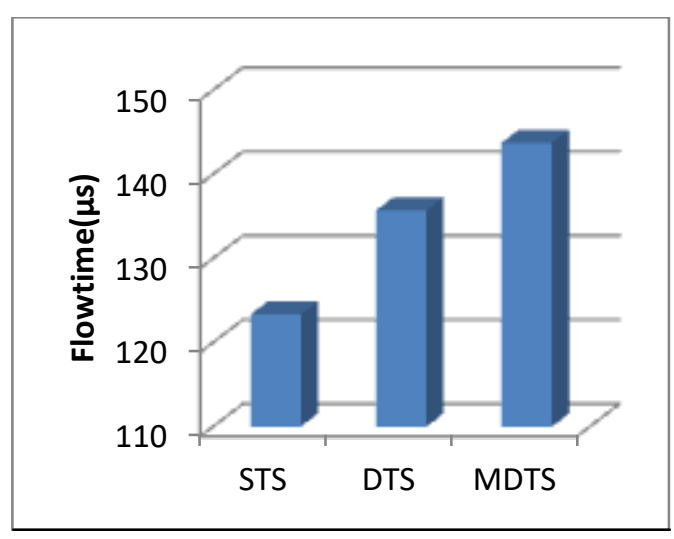

Fig 3. Flowtime for Multiple Heuristics 


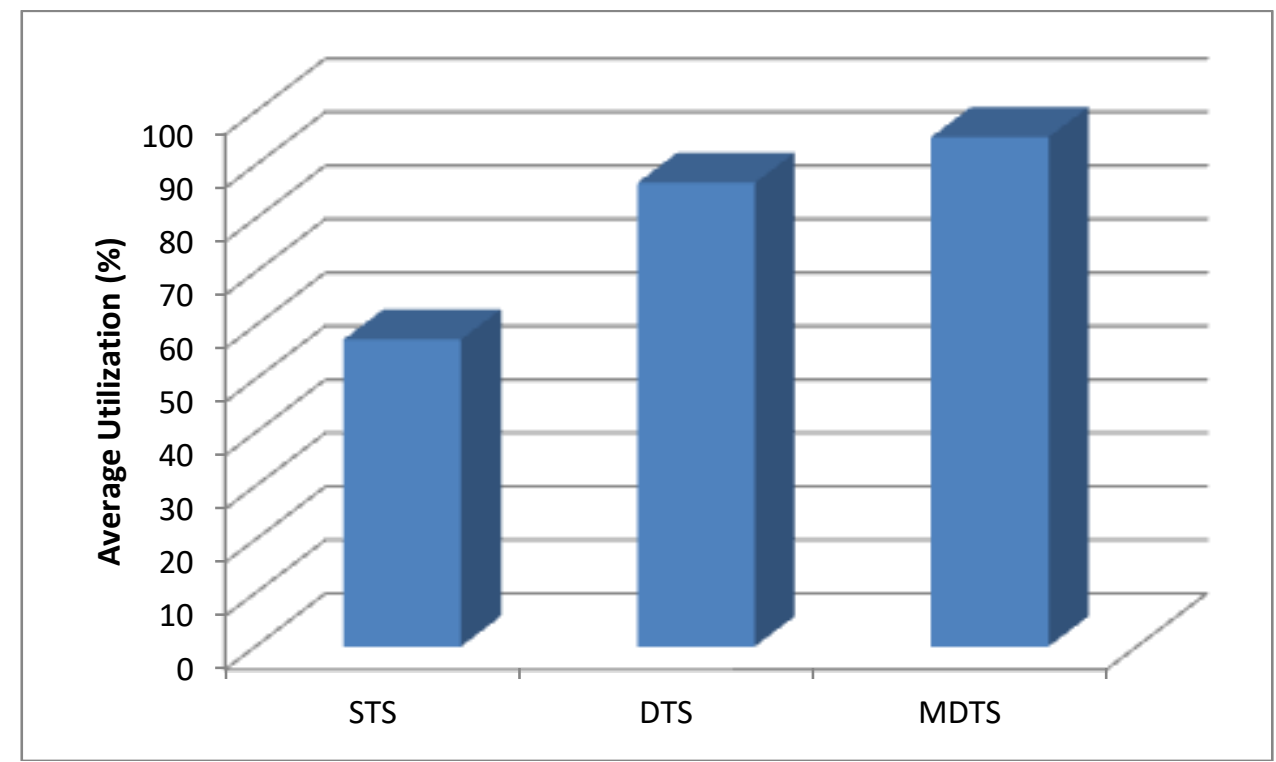

Fig 4. Average Utilization for Multiple Heuristics

After allocation and execution of gridlets each user has certain values of different grid optimization parameters. We are working for multiple users and machines. Each user may have multiple jobs. Each sub task takes some time to execute and based upon task execution rate (MIPS) we have calculated all grid optimization parameters. Proposed strategy is compared with traditional dynamic task scheduling and static task scheduling.

Results show that for every execution of multi objective dynamic task scheduling we got high or sometime equal makespan time, but certainly efficient amount of flowtime. Proposed strategy utilizes resources efficiently as we got good average utilization rate. Certain observations are there that with minimum value of Completion time we got high value of other optimization parameters.

\section{Observation}

1. With adapted grid optimization parameters, we can get minimum task execution time by minimizing makespan time, flowtime and average utilization time of resources.

2. It is also observed that MDTS increased parallelism, the more we able to parallel used resources the better we got task execution rate.

3. We observed that we can get equal utilization from all resources by MDTS. We have tested it for two resources, and we find that all resources are utilized equally so that in such a huge and geographically distributed architecture it is also possible that by MDTS we ensure proper resource utilization.

\section{CONCLUSION}

Computational grid needed large scale simulation that consumes high computing power. Similarly we also need to maintain parallelism among task. The gridsim toolkit supports simulation of multiple tasks to multiple resources that is very beneficial for parallel and cluster computing systems. This would lead to enhance task level parallelism that slightly reduces the resource allocation cost. In order to increased processors capability of resources in computational grid, we need to minimize task execution time. ETC $[\mathrm{t}][\mathrm{m}]$ model is used to calculate and minimize task execution time by minimizing makespan time and flowtime. Proposed implemented approach MDTS using a time shared policy to minimize the resource allocation cost. In MDTS we have multiple users that may have multiple tasks to execute therefore; we are using gridsim to support tasks to resource mapping to increase load balancing among resources and minimizing task execution time.. Simulation study reveals that proposed strategies provides benefits both at 
processors level such as enhanced load distribution and processors utilization, and user level such as minimum job execution time and waiting time for gridlets.

\section{REFERENCES}

I. Reddy, K. H. K., \& Roy, D. S. (2012, March). A hierarchical load balancing algorithm for efficient job scheduling in a computational grid testbed. InRecent Advances in Information Technology (RAIT), $20121^{\text {st }}$ International Conference on (pp. 363-368). IEEE.

II. Salimi, R., Motameni, H., \& Omranpour, H. (2012, December). Task scheduling with Load balancing for computational grid using NSGA II with fuzzy mutation. In Parallel Distributed and Grid Computing (PDGC), 2012 2nd IEEE International Conference on (pp. 79-84).

III. Foster, I., Kesselman, C., \& Tuecke, S. (2001). The anatomy of the grid: Enabling scalable virtual organizations. International journal of high performance computing applications, 15(3), 200-222.

IV. Braun, T. D., Siegel, H. J., Beck, N., Bölöni, L. L., Maheswaran, M., Reuther, A. I., ... \& Freund, R. F. (2001). A comparison of eleven static heuristics for mapping a class of independent tasks onto heterogeneous distributed computing systems. Journal of Parallel and Distributed computing, 61(6), 810-837.

V. Shahid, M., \& Raza, Z. (2014, February). A precedence based distributed job scheduling for computational grid. In Advance Computing Conference (IACC), 2014 IEEE International (pp. 702-707). IEEE.

VI. Krauter, K., Buyya, R., \& Maheswaran, M. (2002). A taxonomy and survey of grid resource management systems for distributed computing. Software: Practice and Experience, 32(2), 135164.

VII. Grzonka, D., Kołodziej, J., Tao, J., \& Khan, S. U. (2015). Artificial Neural Network support to monitoring of the evolutionary driven security aware scheduling in computational distributed environments. Future Generation Computer Systems, 51, 72-86.

VIII. Kokilavani, T., \& Amalarethinam, D. G. (May 2013). EMGEN - A Tool To Create ETC matrix Memory Characteristics For Meta Task Scheduling In Grid Environment, The International Journal of Computer Science \& Applications, 2(3).

IX. Xhafa, F., Barolli, L., \& Durresi, A. (2007, May). Immediate mode scheduling of independent jobs in computational grids. In 21st International Conference on Advanced Information Networking and Applications (AINA'07) (pp. 970-977). IEEE.

X. Wisnesky, R. (2002). Evaluating Scheduling Algorithms on Distributed Computational Grids. In Proceedings of the 11th IEEE Symposium on HighPerformance Distributed Computing.

XI. Bardsiri, A. K., \& Hashemi, S. M. (2012). A comparative study on seven static mapping heuristics for grid scheduling problem. International Journal of Software Engineering and Its Applications, 6(4), 247- 256.

XII. Singh, M., \& Suri, P. K. (2008). QPS Max-Min : a QoS based predictive Max-Min, MinMin switcher algorithm for job scheduling in a grid.Information Technology Journal, 7(8), 1176-1181.

XIII. Wu, M., \& Sun, X. H. (2004, November). Memory conscious task partition and scheduling in grid environments. In Proceedings of the 5th IEEE/ACM International Workshop on Grid Computing (pp. 138- 145). IEEE Computer Society. 
XIV. Xhafa, F., \& Abraham, A. (2010). Computational models and heuristic methods for Grid scheduling problems. Future generation computer systems,26(4), 608-621.

XV. Salimi, R., Motameni, H., \& Omranpour, H. (2012,December). Task scheduling with Load balancing for computational grid using NSGA II with fuzzy mutation.In Parallel Distributed and Grid Computing (PDGC), 2012 2nd IEEE International Conference on (pp. 79-84).

XVI. Buyya, R., Murshed, M.M.: Gridsim: a toolkit for the modeling and simulation of distributed resource management and scheduling for Grid computing. Concurr. Comput.: Pract. Exp. 14, 1175-1220 (2002)

XVII. Ludwig, S. A., \& Moallem, A. (2011). Swarm intelligence approaches for grid load balancing. Journal of Grid Computing, 9(3), 279-301. 